Interactive comment on "Interhemispheric bias in earth's climate response to orbital forcing" by $R$. Roychowdhury and R. M. DeConto

R. Roychowdhury and R. M. DeConto

rajarshi.es@gmail.com

Received and published: 1 May 2016 


\section{Response to Anonymous Referee 1}

Interactive

comment

May 3, 2016

We thank the two anonymous reviewers for providing thorough and constructive feedback on our manuscript. In the following section, we respond to the specific points raised by each reviewer:

\section{Major Comment \#1}

Misleading interpretation. True, the authors correctly point out that what is called the ' $L H B$ ' is in fact an effect of the other hemisphere being different than a mirror. So, for example, when they say that (p.11) "the Southern Ocean has a perennial positive bias", it is in fact a reference to the fact that the northern hemisphere, with its reactive land masses, increases the temperature of the southern hemisphere compared to a situation where the northern hemisphere would be mainly ocean, like the southern hemisphere. . . A "bias" in the northern hemisphere is thus a southern-hemisphere effect; but a "bias" in the northern hemisphere is taken by reference to a land-dominated southern hemisphere. Consequently the biases in the southern and the northern hemisphere are calculated with respect to different references. The authors clarify and defend this approach but it may nevertheless be argued that these subtleties obscure the 
practical implications of these experiments for understanding palaeoclimate records. Consider the following example: "interglacial (warm summer) conditions are muted relative to those of a symmetric earth". At this stage one should already be aware of the fact that the 'symmetric earth' that we use as a reference differs whether we consider the southern or the northern hemisphere. The authors, yet, go on: 'the 'land effect' causes an intensification of 'glacial' vs 'interglacial' conditions in both hemispheres when perihelion coincides with Southern Hemisphere summer". We have the same problem. The following example might make the case more obvious: "At HIGH obliquity, there exists a negative bias on the Northern Hemisphere Continents". This bias is compared to an Earth where southern and northern hemisphere would be dominated by land. Wouldn't we expect a "positive bias" if we were to compare this situation with an earth dominated by oceans?

Author Response: We appreciate the reviewer's careful reading of our manuscript, however, it is not clear to us, what point the reviewer is making. To clarify, the point of the paper is to identify the effect of asymmetry in the hemispheric land distribution of the earth on the climate response of each hemisphere.

When we are calculating the bias for $\mathrm{NH}$, we are trying to identify the effect of southern hemisphere land distribution on Northern Hemisphere climate. We do this by comparing the climate response of $\mathrm{NH}$ in a real world, to a hypothetical symmetrical world, in which $\mathrm{SH}$ is also land dominated. To study the effect of the opposite hemisphere (in this situation, the opposite hemisphere is $\mathrm{SH}$ ), we need to keep the hemisphere in question ( $\mathrm{NH}$ here) constant. By doing the experiments twice, once with real (asymmetric) $\mathrm{SH}$, and once with hypothetical (symmetric) $\mathrm{SH}$, and keeping the $\mathrm{NH}$ land distribution constant in both experiments, we isolate the effect of water dominated $\mathrm{SH}$ on $\mathrm{NH}$ climate response. Similarly, to calculate the bias for SH, we keep the SH land distribution constant in both experiments, and run the simulations twice, once with real (asymmetric) $\mathrm{NH}$, and once with hypothetical (symmetric) $\mathrm{NH}$.

There is no ambiguity in our experiment design, and there exists ONLY ONE possible

Interactive comment
Printer-friendly version

Discussion paper 
symmetrical reference model for each hemisphere. Thus, when we calculate the bias for $\mathrm{NH}$, there can be only one symmetrical reference (North-Symmetric), which can be used to study the effect of $\mathrm{SH}$ on $\mathrm{NH}$ climate. It is not possible to use the SouthSymmetric reference model to calculate the bias in $\mathrm{NH}$, because the first condition, i.e. the hemisphere being considered should be same, is invalidated! Fig 1 and Fig 2 (In the supplement) explains this graphically.

Interactive

comment

The climate response of the Earth at each latitude is different in the Northern and Southern Hemisphere. This difference in the climate response can be broken down into three components:

1. The insolation intensity depending on precession

2. The effect of $\mathrm{NH}$ land configuration on $\mathrm{NH}$ climate response (and vice-versa)

3. The effect of SH land configuration on $\mathrm{NH}$ climate response (and vice-versa)

In our paper, we examine the third point, i.e. the effect of $\mathrm{SH}$ land configuration on $\mathrm{NH}$ climate response, and vice-versa. To achieve our objective, it is imperative that we compare the $\mathrm{NH}$ 'Land Asymmetry Effect' by comparing the $\mathrm{NH}$ climate with a land dominated symmetric earth, and vice versa. Thus, when we say "At HIGH obliquity, there exists a negative bias on the Northern Hemisphere Continents", we are indeed comparing the climate of the Northern Hemisphere with a hypothetical earth in which the Southern Hemisphere is also land dominated, so that we can isolate out the effect which Southern Hemisphere land distribution has on the Northern Hemisphere.

As suggested by Referee 2, we will rephrase the "Land Hemispheric Bias" as "Land Asymmetry Effect". To reiterate, the 'LAE' thus quantifies the effect of the asymmetry in the opposite hemisphere, by systematically comparing the response of the real world, to the response of 1) a symmetric world with mirrored northern hemispheres, and 2) a symmetric world with mirrored southern hemispheres.

"The following example might make the case more obvious: "At HIGH obliquity, there

Printer-friendly version

Discussion paper 
exists a negative bias on the Northern Hemisphere Continents". This bias is compared to an Earth where southern and northern hemisphere would be dominated by land. Wouldn't we expect a "positive bias" if we were to compare this situation with an earth dominated by oceans?"

Again, we'll attempt to clarify. To calculate our 'Effect' (earlier termed 'bias') in the Northern Hemisphere, we change the topography of the Southern Hemisphere. Thus, we compare Northern Hemisphere climate of the 'real world' with a symmetrical reference, which has land dominated SH topography (Fig 1).

1. To estimate our 'Land Asymmetry Effect' for the Northern Hemisphere, we must keep the $\mathrm{NH}$ land topography constant in our comparisons. Thus, we cannot have an ocean dominated Northern Hemisphere if we are trying to estimate the 'Land Asymmetry Effect' for the Northern Hemisphere.

2. If we compare the $\mathrm{NH}$ climate with an earth that has only ocean in the Southern Hemisphere (no land mass, i.e. only ocean), we would get a stronger negative bias, and not a positive bias.

We propose to revise our paper to make our experimental objective clearer to the reader, and replace the usage of the term "Land Hemispheric Bias" with "Land Asymmetry Effect". The practical implications of these results are many, such as the following observation: During the late Pliocene- early Pleistocene, the Northern Hemisphere and Southern Hemisphere integrate summer insolation metrics varied at both obliquity and precessional frequencies. However, the Northern Hemisphere had stronger precessional amplitude than the Southern Hemisphere, which can be ascribed to the "Land Asymmetry Effect" (Manuscript in preparation). 


\section{Major Comment \#2}

CPD

Slab ocean. Slab Ocean is practical to test different land configurations, but ocean heat transport changes are neglected. This may be acceptable for to studying regional effects (monsoon), but the constrain of fixed heat transport becomes uncomfortable when it comes to study inter-hemispheric effects. At the very least caveats had to be given.

Author Response: We agree with the reviewer here, but we think the reviewer might have missed this in the paper. This issue is addressed explicitly in two places in our paper. First, in the methods section where we state: "We use the latest (2012) version of the Global ENvironmental and Ecological Simulation of Interactive Systems (GENESIS) 3.0 GCM with a slab ocean component (Thompson and Pollard, 1997) rather than a full-depth dynamical ocean (Alder et al., 2011). The slab-ocean version of the GCM allows numerous simulations with idealized global geographies and greatly simplifies interpretations of the sensitivity tests by precluding complications associated with ocean model dependencies."

At the end of the paper, we state: "Future work should include complimentary simulations with AOGCMs, to explore the potential modifying role of ocean dynamics on the LHB, not accounted for here."

Furthermore, the actual ocean heat transport in the slab component is not fixed, but changes relative to the land-ocean fraction in each band of latitude and as a function of the local temperature gradient. While slab-ocean GCMs clearly have limitations, the use of a fully coupled AOGCM would be computationally impractical for this exercise, and would add additional, likely model-dependent, complexities to this first analysis of the LHB issue.
Interactive comment

Printer-friendly version

Discussion paper 
The focus on temperature response obscures the interest of using an advanced general circulation model for this study. It seems that most if not all the conclusions presented here would have been similarly obtained with an Energy Balance Model. Nothing is said about atmospheric connections, hydrological budget, and pressure response. Possible implications for moisture transport are evoked in the conclusions but they are not backed by proper analysis.

Author Response: : We agree with the referee in principle. However, we use a GCM in this study, because it allows a more realistic spatial analysis (including the influence of atmospheric dynamics) that would be precluded by simple EBMs. We view the fact that the general conclusions would likely be supported by a simple EBM as a good thing, supporting the veracity of the overall conclusions. We also note, that EBMs have been replaced as the standard tool for the modern paleoclimatologists in data-model comparison exercises. One goal of this paper is to provide some guidance on the strength of the LHB, when interpreting paleoclimate data on orbital timescales.

We feel that an in-depth dynamical analysis is beyond the scope of this initial investigation of the issue. However, some simple analysis of orbital controls on meridional moisture transports and precipitation could be added in a revised manuscript.

\section{Minor Comments}

p. 4 line 19 : "should theoretically move equally north and south according to the C3 CPD Interactive comment Printer-friendly version Discussion paper hemisphere experiencing summer". The theory precisely says that it should not be symmetric! (given the asymmetry) 
Author Response: We agree. What we meant was "should ideally move equally north and south according to the hemisphere experiencing summer". We will correct this in future.

p. 10 line 15 : "perennial positive bias" : in what sense is it perennial ?

Author Response: Perennial positive bias refers to positive bias present at all orbital configurations, i.e. at all obliquity and precessional configurations.

1) Use standard definitions when they exist. E.g.: "orbital precession" is in fact the heliocentric longitude of the perihelion.

Author Response: We agree that the standard definition should be used instead.

\section{References to Literature}

Recent landmark citations (Huybers (2006), Raymo (2006)) are valid, but proper credit should be given to Pollard for the use of PDD, and Berger for pioneering analysis of insolation.

Author Response: We will cite Pollard in our revised paper. We have cited Berger in our paper.

The introduction quotes earlier works on effects associated with Earth's geography on climate sensitivity, but little is said about how the results presented here compare with earlier findings.

Author Response: The climate response of the Earth at each latitude is different in the Northern and Southern Hemisphere. This difference in the climate response can be broken down into three components:

1. The insolation intensity depending on precession 2 . The effect of $\mathrm{NH}$ land configu- 
ration on $\mathrm{NH}$ climate response (and vice-versa) 3. The effect of $\mathrm{SH}$ land configuration on $\mathrm{NH}$ climate response (and vice-versa)

CPD

The earlier works, which have been cited in the introduction, refer to the 1st and 2nd points, or the effect of insolation and Earth's local geography on climate sensitivity. In our study, we focus on the 3rd point, i.e. the effect of the geography of the opposite hemisphere on climate sensitivity. The results communicated here are different from any kind of previous study, and hence we have not compared our results with earlier findings. 